\title{
EPIDEMIOLOGIA DE HELMINTOS GASTRINTESTINAIS EM BÚFALOS
}

\section{EPIDEMIOLOGY OF GASTROINTESTINAL HELMINTHS IN BUFFALOES}

\author{
Daniele Bier ${ }^{1}$ \\ Letícia e Silva Teruya² \\ Dyego Gonçalves Lino Borges² \\ Juliana Paniago Lordello Neves ${ }^{2}$ \\ Larissa Berreza dos Santos ${ }^{2}$ \\ Fernando de Almeida Borges ${ }^{2^{*}}$ \\ ${ }^{1}$ Universidade Católica Dom Bosco, Campo Grande, MS, Brasil. \\ ${ }^{2}$ Universidade Federal de Mato Grosso do Sul, Campo Grande, MS, Brasil. \\ *Autor para correspondência - fernando.borges@ufms.br
}

\section{Resumo}

Este trabalho objetivou avaliar a prevalência e a sazonalidade da infecção natural por parasitos gastrintestinais em bubalinos de aptidão leiteira de diferentes categorias, no município de Bandeirantes, Mato Grosso do Sul. Foram avaliados 41 búfalos da raça Murrah, divididos em três categorias, sendo 11 lactentes, com idade de 0 a 8 meses, 10 desmamados, de 8 a 18 meses, e 20 fêmeas adultas com idade superior a 18 meses. Os animais adultos apresentaram-se livres de parasitismo ou com carga parasitária muito baixa. A categoria lactente apresentou pico de contagem de ovos por grama de fezes (OPG=2038) em novembro, quando a precipitação acumulada foi de 69,3 $\mathrm{mm}$ e a temperatura $25,95{ }^{\circ} \mathrm{C}$, enquanto a menor contagem de ovos (167) ocorreu em maio, dois meses após pico de $234,68 \mathrm{~mm}$ e $20,59{ }^{\circ} \mathrm{C}$. Os búfalos desmamados apresentaram dois picos de $\mathrm{OPG}$, de 1288 e 875 , em setembro e junho, com precipitação de 41,45 e 18,4 mm e temperatura de 24,16 e $20,69^{\circ} \mathrm{C}$, respectivamente. Foi observada correlação significativa $(\mathrm{p}=0,01)$ e negativa $(\mathrm{r}=-0,33)$ entre o OPG dos bezerros lactentes e precipitação pluviométrica. Os parasitos gastrintestinais mais frequentes foram Haemonchus sp. e Cooperia sp.

Palavras-chave: bubalinos; Cooperia; Eimeria; Haemonchus; sazonalidade.

\begin{abstract}
This study evaluated the prevalence and seasonality of natural infection by gastrointestinal parasites and the susceptibility of different dairy buffalo categories in Bandeirantes, state of Mato Grosso do Sul, Brazil. Forty-one Murrah buffaloes were divided into three categories, 11 suckling calves (0-8 months old), 10 weaned calves (8-18 months old), and 20 adult females over the age of 18 months. Adult animals were free from parasitism or showed very low parasite load. The suckling calves showed a peak of egg per gram of feces $(\mathrm{EPG}=2038)$ in November, when the average rainfall was $69.3 \mathrm{~mm}$ and the temperature $25.95{ }^{\circ} \mathrm{C}$, while the lowest egg count (167) occurred in May, two months after the peak of $234.68 \mathrm{~mm}$ and $20.59{ }^{\circ} \mathrm{C}$. Weaned calves showed two peaks of EPG from 1288.89 and 875 in September and June, with rainfall of 41.45 and $18.4 \mathrm{~mm}$, temperature 24.16 and $20.69^{\circ} \mathrm{C}$, respectively. Significant $(\mathrm{p}=0.01)$ and negative $(\mathrm{r}=-0.33)$ correlation between the EPG of suckling calves and precipitation was observed. The most common gastrointestinal parasites were
\end{abstract}


Haemonchus sp. and Cooperia sp.

Keywords: bubalines; Cooperia; Eimeria; Haemonchus; seasonality.

Recebido em: 20 de abril de 2016

Aceito em: 19 janeiro de 2018 .

\section{Introdução}

O búfalo (Bubalus bubalis) é uma espécie importante como fonte de matéria prima para laticínios, carne e adubo em diversos países. A vida útil de búfalos é maior do que a de bovinos, geralmente mais de 17 anos, chegando até 25 anos de idade. Devido a sua excelente capacidade de tração, em alguns países os búfalos são chamados de "tratores vivos"(1). Porém, o conhecimento científico sobre esse animal não é compatível com o aumento de seu número e importância. A FAO, Organização das Nações Unidas para Alimentação, classificou a bovinocultura na Ásia como um bem de produção importante, contudo desvalorizado ${ }^{(2)}$.

A bubalinocultura é uma atividade em expansão no Brasil devido à adaptação desses animais ao ambiente e ao aumento no consumo de seus produtos, especialmente de mussarela de búfala ${ }^{(3)}$. $\mathrm{O}$ rebanho bubalino brasileiro está estimado em 1,185 milhão de animais, com 4,6\% na região Centrooeste. No Mato Grosso do Sul há cerca de 16 mil búfalos ${ }^{(4)}$.

Apesar de serem considerados animais rústicos, conhecidos por apresentarem grande resistência ao desenvolvimento de doenças comuns aos bovinos, os bubalinos são susceptíveis às infecções parasitárias, principalmente pelo hábito de permanecerem aglomerados por longos períodos, o que pode ser agravado por condições nutricionais precárias e confinamento com alta taxa de lotação ${ }^{(1,5)}$.

A parasitose é um dos fatores que interfere no desenvolvimento da pecuária bovina e bubalina e ocupa grande destaque por ocasionar perdas econômicas relacionadas à baixa produtividade do rebanho, retardamento do desenvolvimento dos animais, morte e gastos excessivos com manejo e medicamentos $^{(6)}$. Para reduzir o efeito do parasitismo na produção animal, é de fundamental importância conhecer a biologia dos parasitos dos animais da região ${ }^{(7)}$.

As condições climáticas sobre os estágios de vida livre, como a precipitação pluviométrica e as faixas climáticas favoráveis, regulam as mudanças sazonais na dinâmica das populações dos parasitos ${ }^{(8,9)}$. Além disso, a interação hospedeiro-parasito pode ser influenciada pelas características das pastagens, fatores genéticos, concentração de animais por área, faixa etária e nutrição ${ }^{(10,11)}$.

Pouco se conhece sobre a epidemiologia dos parasitos gastrintestinais em búfalos criados a pasto em regiões de cerrado brasileiro. Nesse contexto, o objetivo do presente trabalho foi estudar a etiologia, sazonalidade e susceptibilidade ao parasitismo em diferentes categorias de búfalos criados em sistema de pastejo rotacionado em Mato Grosso do Sul.

\section{Material e métodos}

O trabalho foi realizado entre setembro de 2010 e julho de 2011, em uma propriedade rural, localizada no município de Bandeirantes, latitude $5432852^{\circ} \mathrm{O}$ e longitude $20.01401^{\circ} \mathrm{S}$, no estado de Mato Grosso do Sul. 
Foram utilizados 41 búfalos da raça Murrah, divididos em três categorias: a) 11 bezerros lactentes, com idade de 0 a 8 meses; b) 10 bezerros desmamados, de 8 a 18 meses; c) 20 fêmeas adultas, com idade acima de 18 meses.

Cada lote era mantido separado dos demais em pastagens de Brachiaria brizantha cv. Marandu em sistema de pastejo rotacionado. Os bubalinos não tinham contato com outra espécie ruminante doméstica na propriedade.

A cada 28 dias, foram coletadas amostras de fezes diretamente da ampola retal, com sacolas plásticas, individualmente, para a realização de exames de contagem de ovos por grama de fezes (OPG), segundo a técnica de Gordon \& Whitlock $^{(12)}$ modificada, com sensibilidade de 1:50. Para a identificação dos gêneros de estrongilídeos, foram realizadas coproculturas ${ }^{(13)}$.

Os dados de precipitação pluvial e de temperatura diárias foram obtidos pela estação meteorológica n83611 do Instituto Nacional de Pesquisas Espaciais (INPE), localizada a $58 \mathrm{~km}$ do local do estudo.

Os resultados do OPG mostraram uma distribuição normal. A comparação entre as médias de OPG de bezerros lactentes e desmamados foi realizada pela Análise de Variância (ANOVA) e teste T com significância de 95\%. Para avaliar a correlação entre o parasitismo e as condições climáticas, foi realizado o teste de normalidade de D’Agostino e Pearson. Após o teste, foi calculado o coeficiente de correlação de postos de Spearman. Utilizou-se o programa estatístico GraphPad Prism version 5.2 for Windows (GraphPad Software, San Diego, California, USA, www.graphpad.com).

\section{Resultados e discussão}

No presente trabalho foram encontrados os seguintes parasitos em búfalos: Haemonchus sp, Cooperia sp., Trichostrongylus sp. e Oesophagostomum sp. Os gêneros Strongyloides sp. e Eimeria sp. foram encontrados, no OPG, no entanto, com frequência e intensidade menores. Mesmo nos animais jovens, não foi encontrado Toxocara vitulorum, que é considerado o nematódeo mais patogênico para essa categoria e pode ser encontrado em altas taxas de prevalência no Brasil ${ }^{(14)}$.

No período compreendido entre setembro de 2010 e julho de 2011, a precipitação pluviométrica acumulada na região de estudo foi de $89,77 \mathrm{~mm}( \pm 12,64)$ e a temperatura do ar apresentou pouca variação, com média de $23,94{ }^{\circ} \mathrm{C}( \pm 1,74)$. As menores temperaturas médias registradas foram em maio e junho, em torno de $16^{\circ} \mathrm{C}$. A temperatura durante todo o período do estudo não teve efeito limitante no desenvolvimento dos parasitas no meio ambiente. Não houve correlação significativa $(\mathrm{P}>0,05)$ entre temperatura média e as médias de OPG nas categorias bezerros lactentes e desmamados. Porém, a precipitação pluviométrica apresentou variação mais relevante e foi o fator externo adotado para correlacionar à população de parasitos. Araújo e Lima ${ }^{(15)}$ também relataram que a temperatura não teve efeito limitante no desenvolvimento de helmintos gastrointestinais nas pastagens, ao contrário da precipitação pluvial, que delimitou períodos distintos no número de helmintos recuperados.

Dos 21 bezerros bubalinos estudados, todos foram positivos para diferentes parasitas durante o período de estudo, indicando uma alta incidência e sem diferença $(\mathrm{P}=0,1139)$ de susceptibilidade entre as categorias lactente e desmamado (Figura 1). Isso pode se atribuir ao fato de búfalos serem mais propensos a parasitas internos devido a seu hábito de chafurdar em áreas pantanosas, de onde eles podem ter engolido os ovos/larvas de diferentes espécies de parasitos ${ }^{(16)}$. Bhutto et al. ${ }^{(17)}$, ao 
estudarem helmintos gastrointestinais em bezerros bubalinos no Paquistão, encontraram uma prevalência de $47 \%$. Esses autores consideram a idade dos animais como um fator importante na prevalência de infecções por helmintos. Soulsby ${ }^{(18)}$ e Taylor et al. ${ }^{(19)}$ também documentaram maior prevalência em bezerros jovens e sua ocorrência relacionada à infecção dos pais ou com a transferência de larvas L3 no colostro, leite e infecção pós-natal devido às más condições de higiene.

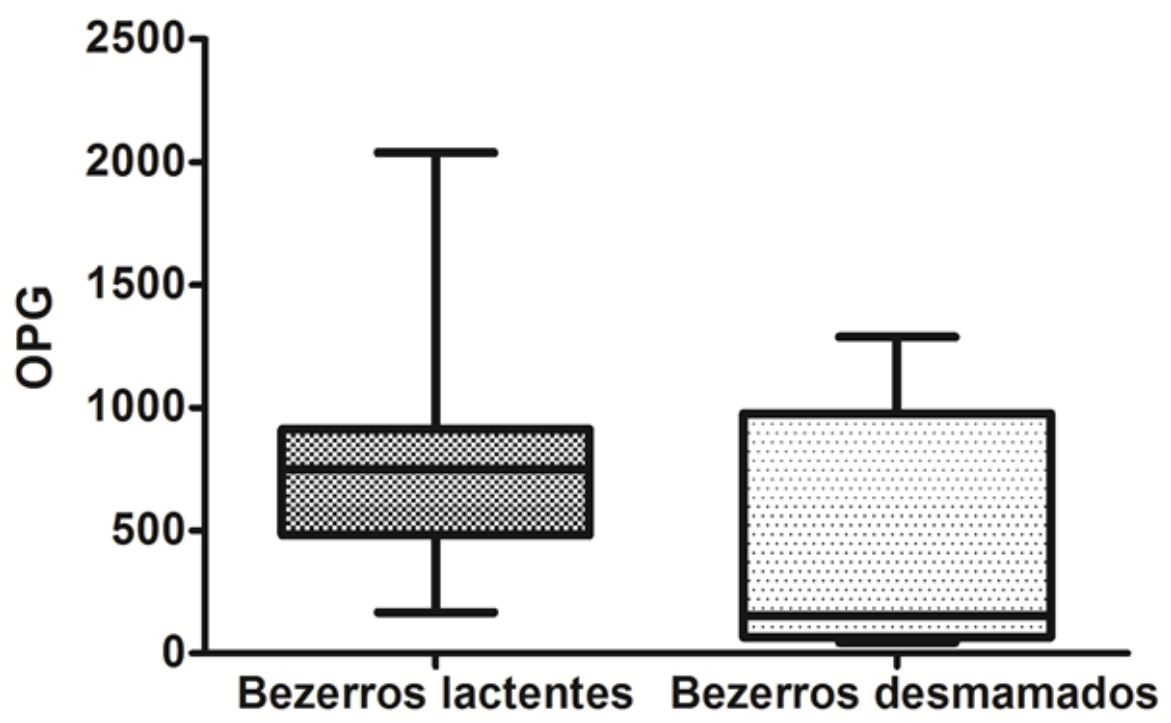

Figura 1. Médias (dp) de OPG de bezerros bubalinos desmamados e lactentes. Bandeirantes, MS.

Dos 20 animais pertencentes à categoria adulta, 13 apresentaram-se livres de parasitos gastrintestinais e a média de OPG dos sete animais positivos foi de 142. Portanto, é uma categoria que apresenta elevada resistência à verminose.

A categoria lactente apresentou pico de contagem de ovos por grama de fezes (OPG=2038) em novembro, quando a precipitação acumulada foi de $69,3 \mathrm{~mm}$ e temperatura $25,95{ }^{\circ} \mathrm{C}$, enquanto a menor contagem de ovos $(\mathrm{OPG}=167)$ ocorreu em maio, dois meses após o pico de precipitação pluviométrica $(234,68 \mathrm{~mm}$ ) (Figura 2). Os búfalos desmamados apresentaram dois picos de OPG (1288 e 875), em setembro e junho, com precipitação de 41,45 e 18,4 mm, respectivamente (Figura 2). Nesta categoria, entre fevereiro e maio, o valor de OPG manteve-se reduzido, apresentando a menor contagem de ovos $(44,44)$ em maio, quando a precipitação foi de $9,5 \mathrm{~mm}$. Foi observada correlação significativa $(\mathrm{p}=0,01)$ e negativa $(\mathrm{r}=-0,33)$ entre o OPG dos bezerros lactentes e a precipitação pluviométrica, enquanto o mesmo não ocorreu com animais desmamados $(\mathrm{p}=0,053)$. A redução do OPG nos desmamados talvez seja mais influenciada pela resposta imune com o aumento da idade do animal do que pelos efeitos climáticos. 


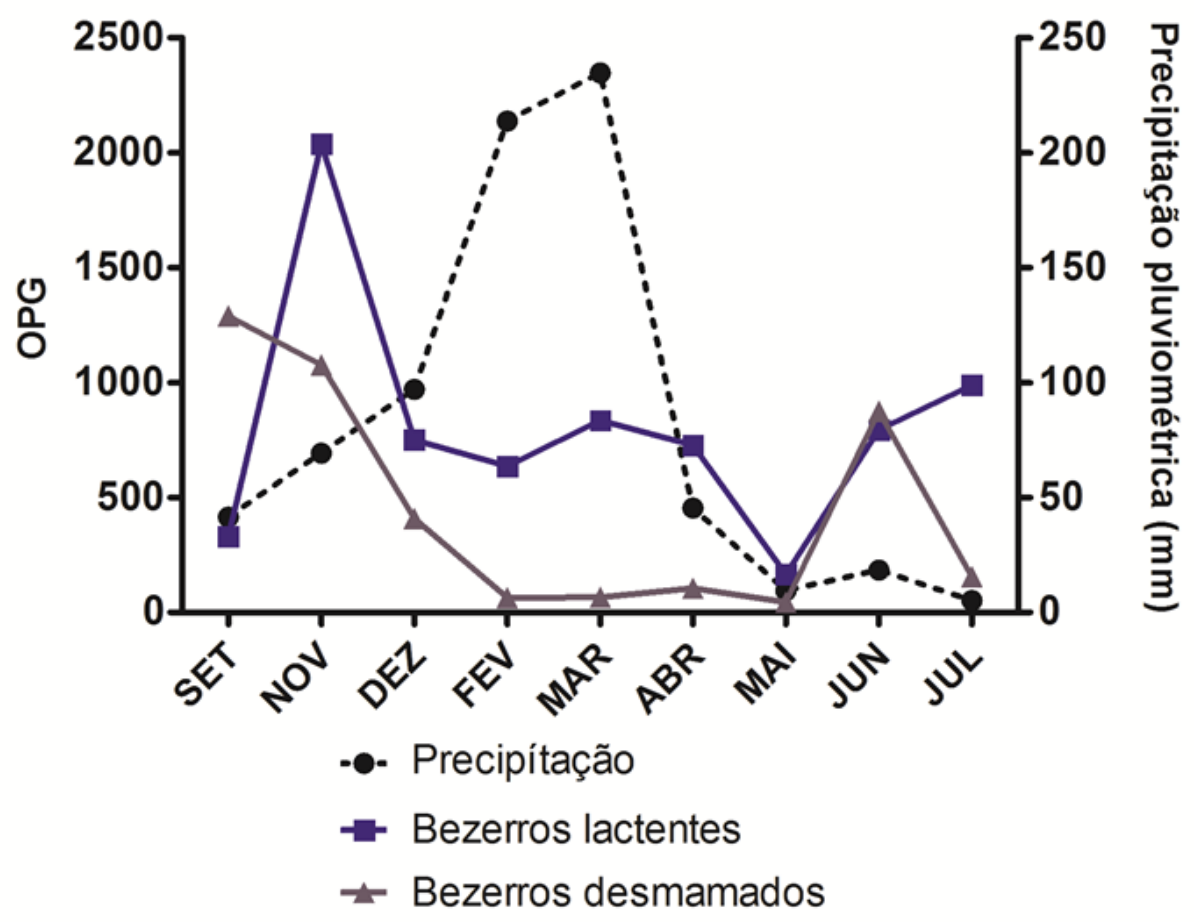

Figura 2. Médias de OPG de bezerros bubalinos lactentes e desmamados e precipitação pluviométrica no período de setembro de 2010 a julho de 2011. Bandeirantes, MS.

Quando verificada a relação dos valores médios das contagens de OPG de helmintos da ordem Strongylidea, de bezerros lactentes, observou-se que um maior número de ovos foi encontrado durante o período seco. Correlação negativa entre os valores de OPG nos animais lactentes e a precipitação pluviométrica foi também verificada por Bastianetto et al. ${ }^{(20)}$ em Minas Gerais e por Láu ${ }^{(21)}$ no Pará, os quais, ao estudarem a variação da quantidade de ovos de parasitas presentes nas fezes de bezerros búfalos, observaram os picos de OPG no início e no final do período seco (meses de junho-agosto e novembro-janeiro).

Diferentemente do presente estudo, Khalil-ur-Rehman et al. ${ }^{(16)}$, estudando nematódeos gastrintestinais em búfalos no Paquistão, informaram que a carga total de vermes nos animais foi maior durante a estação chuvosa e menor durante a estação seca.

Essa variação do número de OPG durante o ano está relacionada à menor quantidade ou à ausência de larvas infectantes em determinado período ou à alteração na susceptibilidade do hospedeiro. Isso pode ser provado pelas condições ambientais desfavoráveis para o desenvolvimento, eclosão e migração das larvas infectantes ${ }^{(22)}$. Além disso, Bastianetto e Leite ${ }^{(23)}$ relataram, que, por ser uma espécie poliéstrica estacional de dia curto, a concentração de nascimentos de búfalos ocorre no término do período de verão e a desmama no final da primavera, coincidindo com os meses que apresentam alta disponibilidade de larvas de nematoides infectantes nas pastagens.

Nas coproculturas dos bezerros, foram recuperadas larvas de Haemonchus sp., Cooperia sp., Trichostrongylus sp. e Oesophagostomum sp, sendo os dois primeiros os endoparasitas mais frequentes (Figuras 3 e 4). Liu et al. ${ }^{(24)}$, ao avaliarem búfalos no Paquistão, também encontraram maior número de Haemonchus contortus, com uma prevalência de $61,8 \%$.

Haemonchus apresentou maior frequência em setembro, novembro e dezembro, quando a precipitação pluviométrica estava baixa, e diminuição durante o período chuvoso, enquanto Cooperia 
apresentou o pico no mês de fevereiro e se manteve alta nos meses subsequentes, de alta precipitação pluviométrica. Larvas de Trichostrongylus e Oesophagostomum foram recuperadas em baixo número durante o experimento (Figuras 3 e 4 ).

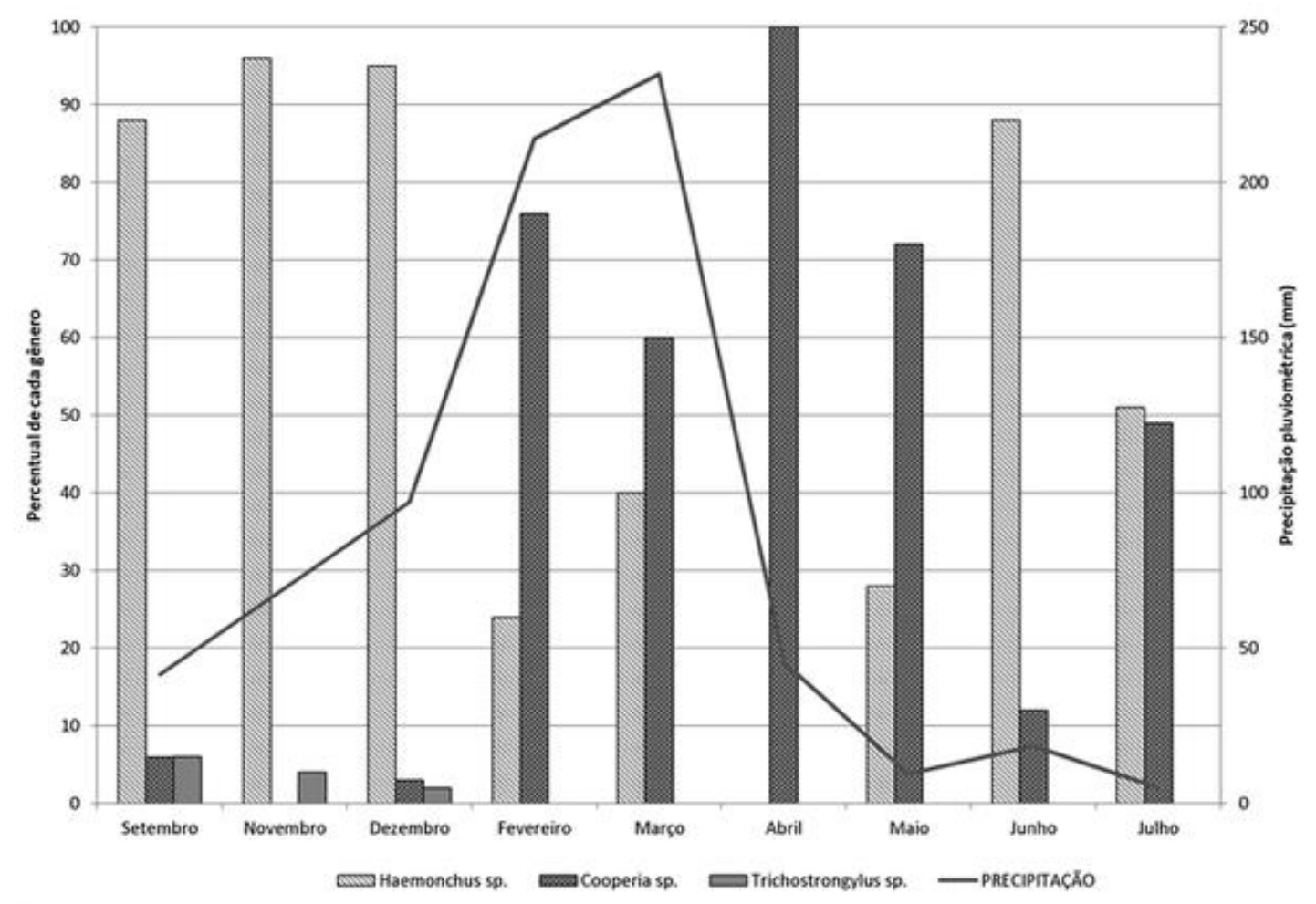

Figura 3. Ocorrência de larvas L3 em coproculturas de bezerros bubalinos lactentes em relação à precipitação no período de setembro de 2010 a julho de 2011.

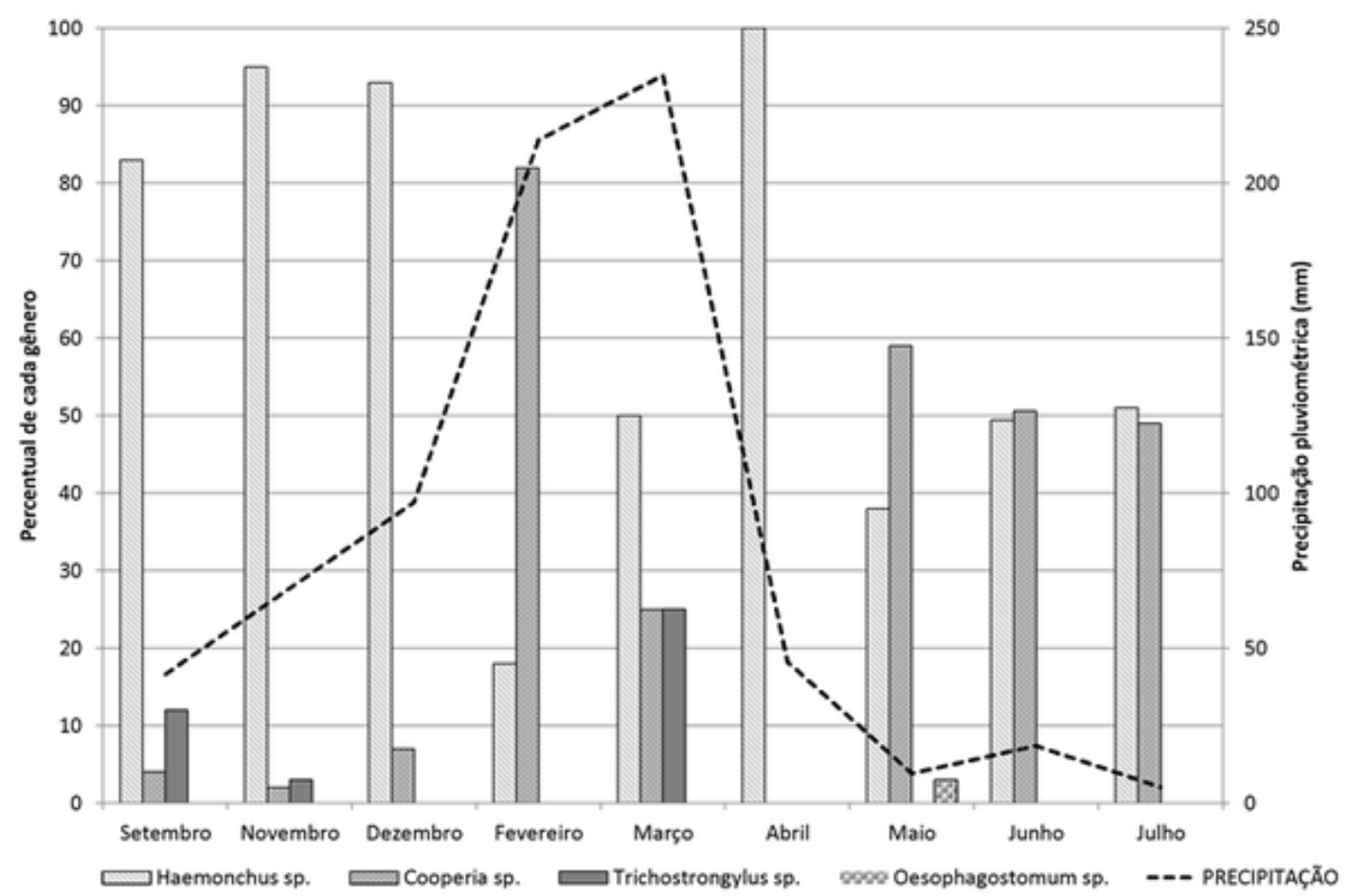

Figura 4. Ocorrência de larvas L3 em em coproculturas bezerros bubalinos desmamados em relação à precipitação no período de setembro de 2010 a julho de 2011. 
Mamun et al. ${ }^{(25)}$ relataram que a flutuação sazonal do ano tem um efeito significativo sobre a prevalência de infecções parasitárias gastrointestinais em búfalos. Diferentemente do presente estudo, observaram infecção relativamente maior por parasitas gastrointestinais na estação chuvosa (71,70\%), seguido de verão $(58,90 \%)$ e inverno $(52,27 \%)$. Já no trabalho realizado por Starke et al. ${ }^{(26)}$, também no Mato Grosso do Sul, avaliando o desenvolvimento de ovos e larvas de estrongilídeos em búfalos jovens, esses autores concluíram que nos períodos secos houve uma maior permanência dos ovos nas massas fecais sem a evolução para larvas infectantes.

\section{Conclusão}

Os parasitos gastrintestinais mais frequentes em bezerros bubalinos em Bandeirantes, no Mato Grosso do Sul, foram os helmintos Haemonchus sp. e Cooperia sp. As categorias bezerro lactente e bezerro desmamado são susceptíveis à verminose, enquanto animais com idade superior a 18 meses apresentam parasitismo leve ou inexistente. As maiores cargas parasitárias na região do estudo podem ser esperadas no início e no final da estação da seca.

\section{Referências}

1. Cockrill WR. O búfalo em ascensão: animal doméstico fundamental, criação, proteção e saúde animal. In: Ramos AA, Villares JB, Moura JC. (Ed.). Os búfalos. Piracicaba: Fundação de Estudos Agrários Luiz de Queiroz, 1981.p. 01-54.

2. FAO - Food and Agriculture Organization. Water buffalo: an asset undervalued. FAO Regional Office for Asia and the Pacific; Bangkok, Thailand: 2000. Disponível em: http://cdn.aphca.org/dmdocuments/APHCA\%20Publications/w buffalo.pdf. Inglês

3. ABCB - Associação Brasileira de Criadores de Búfalos. ABCB trabalha para melhorar o mercado de búfalos no Brasil. Acessado em 23 jul. 2014. Online. Disponível em: http://www.bufalo.com.br.

4. IBGE - Instituto Brasileiro de Geografia e Estatística. Produção da Pecuária Municipal, v. 38, 201. $\begin{array}{llllll}\text { Acessado em } & 14 & \text { nov. } & \text { Disponível }\end{array}$ http://www.ibge.gov.br/home/estatistica/economia/ppm/2010/tabelas_pdf/tab03.pdf.

5. Láu HD. Doenças em Búfalos no Brasil: Diagnóstico, Epidemiologia e Controle. Belém: EMBRAPA, CPATU, $1999 . \quad$ p. 27-31. Disponível em: https://www.bdpa.cnptia.embrapa.br/consulta/busca?b=pc\&id=388051\&biblioteca=vazio\&busca=14940\&qF acets $=14940 \&$ sort $=\&$ paginacao $=t \&$ paginaAtual $=1$.

6. Lima WS. Controle das helmintoses dos bovinos. In: BRESSAN, M. Práticas de manejo sanitário em bovinos de leite. Juiz de Fora: EMBRAPA Gado de Leite, 2000. 65p. Disponível em: https://www.embrapa.br/gado-de-leite/busca-de-publicacoes/-/publicacao/594326/praticas-de-manejosanitario-em-bovinos-de-leite.

7. Furlong J, Abreu HGL, Verneque RS. Parasitoses dos bovinos na região da Zona da Mata de Minas Gerais: I. Comportamento estacional de nematódeos gastrintestinais. Pesquisa Agropecuária Brasileira, 1985; 20: 143153. Disponível em: http://seer.sct.embrapa.br/index.php/pab/article/view/15002.

8. Lima WS, Fakuri E, Guimarães MP, Malacco MA. Dinâmica das helmintoses de bovinos de leite na região Metalúrgica de Minas Gerais. Revista Brasileira de Parasitologia Veterinária, 1997; 6: 97-103. Disponível em: 
http://www.cbpv.org.br/rbpv/documentos/621997/c6297_103.pdf.

9. Ungerfeld R \& Correa O. Social dominance of female dairy goats influences the dynamics of gastrointestinal parasite eggs. Applied Animal Behaviour Science, 2007; 105(4): 249-253. Disponível em: http://www.sciencedirect.com/science/article/pii/S0168159106001584. Inglês.

10. Gibbs HC. Transmission of parasites with reference to the strongyles of domestic sheep and cattle. Canadian Journal of Zoology, 1973; 51: 281-289. Disponível em: http://www.nrcresearchpress.com/doi/abs/10.1139/z73-040\#.VxeBgUePXEk. Inglês

11. Catto JB. Desenvolvimento e sobrevivência de larvas infectantes de nematódeos gastrintestinais de bovinos, durante a estação seca, no Pantanal Mato-Grossense. Pesquisa Agropecuária Brasileira, 1982; 17(6): 923-927. Disponível em: http://seer.sct.embrapa.br/index.php/pab/article/view/15948.

12. Gordon HM. \& Whitlock HV. A new technique for counting nematode eggs in sheep feces. Journal of the Council for Scientific and Industrial Research, 1939; 12: 50-52. Disponível em: https://publications.csiro.au/rpr/download?pid=procite:21259a33-8a8e-4add-9315-

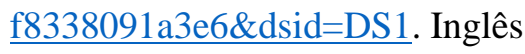

13. Roberts FH \& O'sullivan PJ. Methods for egg counts and larval cultures for strongyles infesting the gastrointestinal tract of catle. Australian Journal of Agricultural Research, 1950; 1: 99-102. Disponível em: http://www.publish.csiro.au/paper/AR9500099.htm.

14. Barbieri, F. S. ; Brito, L. G. ; Figueiró, M.R. ; Bandeira, P. F. ; Lanzoni, M. M. ; Nascimento, A. X.. Controle de Toxocara vitulorum em búfalos jovens em Presidente Médici, Rondônia, Brasil. Porto Velho: Embrapa Rondônia, 2010 (Comunicado Técnico 357). Disponível em: http://www.infoteca.cnptia.embrapa.br/infoteca/handle/doc/884337

15. Araujo RN, Lima WS. Infecções helmínticas em um rebanho leiteiro na região Campo das Vertentes de Minas Gerais. Arquivo Brasileiro de Medicina Veterinária e Zootecnia, 2005; 57(2): 186-193. Disponível em: http://dx.doi.org/10.1590/S0102-09352005000800009.

16. Khalil-ur-Rehman KJ, Tunio MT, Kuthu H. Passive surveillance of gastrointestinal parasites in bufflaoes of Mandi Bahauddin and Gujrat districts of the Punjab. The Journal of Animal \& Plant Sciences, v. 19, n. 1, p. 17-19, 2009. Disponível em: http://www.thejaps.org.pk/docs/19-no-1-2009/09-901.pdf. Inglês

17. Bhutto B, Phullan MS, Rind R, Soomro AH. Prevalence of Grastro-Intestinal Helminths in Buffalo Calves. Online Journal of Biological Sciences, 2002; 2(1): 43-45. Disponível em: http://www.scialert.net/abstract/?doi=jbs.2002.43.45. Inglês.

18. Soulsby EJL. Helminths, Arthropods and Protozoa of Domesticated Animals. 7 th Ed. Lea \& Febiger, Philadelphia, 156-239.

19. Taylor MA, Coop RL, Wall RL. Veterinary Parasitology, $3^{\text {rd }}$ Ed. Wiley-Blackwell, London, UK, 2007, 600p. Inglês.

20. Bastianetto E, Costa JO, Guimarães MP, Frei CMV. Population Dynamic, Anthelmintic Treatments and the Influence of Helminth Infections on Weight Gain in Water Buffalo Calves (Bubalus Bubalis). Journal of Buffalo Science, 2012; 1: 5-12. Disponível em: http://lifescienceglobal.com/pms/index.php/JBS/article/view/161. Inglês.

21. Láu HD. Helmintoses gastrintestinais dos bufalinos no estado do Pará: epidemiologia e controle. Belém: EMBRAPA, $\quad$ CPATU, $1993 . \quad 38 \quad$ p. $\quad$ Disponível em: https://www.infoteca.cnptia.embrapa.br/bitstream/doc/378468/1/CPATUDoc72.pdf.

22. Starke WA \& Machado ZR. Helmintíases em Búfalos I - Desenvolvimento de ovos e larvas de estrongilídeos parasitas de búfalos jovens, no Município de Selvíria, MS, nas estações secas e chuvosas. 
Arquivo Brasileiro de Medicina Veterinária e Zootecnia, 1991; 43(4): 315-327. Disponível em: http://www.bvs-vet.org.br/pt/vetindex/29656/helmintiases-em-bufalo-i-desenvolvimento-de-ovos-e-larvasde-estrongilideos-parasitas-de/.

23. Bastianetto E \& Leite RC. Aspectos epidemiológicos e controle das doenças parasitárias em bubalinos. Ciência Animal Brasileira, 2009; 1: 1-17. Disponível em: http://revistas.ufg.emnuvens.com.br/vet/article/viewFile/7666/5439.

24. Liu Y, Li F, Liu W, Dai RS, Tan YM, He DS, Lin RQ, Zhu XQ. Prevalence of helminths in water buffaloes in Hunan Province, China. Tropical Animal Health Production, 2009; 41: 543-546. Disponível em: http://link.springer.com/article/10.1007\%2Fs11250-008-9219-1\#page-1.

25. Mamun MAA, Begum N, Mondal MMH. A coprological survey of gastro-intestinal parasites of water buffaloes (Bubalus bubalis) in Kurigram district of Bangladesh. Journal of the Bangladesh Agricultural University, 2011; 9(1): 103-109. Disponível em: http://www.banglajol.info/index.php/JBAU/article/view/8752.

26. Starke WA, Zocoller MC, Machado RZ, Montenegro EL. Helmintíases em Búfalos II - Sobrevivência de larvas de nematódeos parasitos de búfalos jovens nas fezes depositadas em pastagens no município de Selvíria, MS, nos períodos secos e chuvosos. Revista Brasileira de Parasitologia Veterinária, 1992; 1(1): 7-15. Disponível em: http://www.cbpv.org.br/rbpv/documentos/111992/c1107_15.pdf. 